\title{
15 OBSERVATIONS FROM A FIELD STUDY ON DEVELOPING A FRAMEWORK FOR PRE-USAGE EVALUATION OF CASE TOOLS
}

\author{
Adam Rehbinder \\ Department of Computer Science \\ University of Skövde \\ SE-521 28 Skövde \\ Sweden \\ Brian Lings \\ Department of Computer Science \\ University of Exeter \\ Exeter EX4 4PT \\ England \\ Björn Lundell \\ Department of Computer Science \\ University of Skövde \\ SE-521 28 Skövde \\ Sweden \\ Runo Burman \\ Common Skills and Methods \\ Volvo IT \\ SE-40508 Göteborg \\ Sweden \\ Anette Nilsson
Operations Center
Volvo IT
SE-541 87 Skövde
Sweden
}

\begin{abstract}
In this paper, we address the issue of method validation by means of method transfer. We report on experiences from an
\end{abstract}

The original version of this chapter was revised: The copyright line was incorrect. This has been corrected. The Erratum to this chapter is available at DOI: 10.1007/978-0-387-35489-7_33 
application of a proposed method for evaluation framework development in a field study, and demonstrate that the method user, previously unfamiliar with the method, was able to successfully apply it in a complex setting.

From post project interviews it was evident that stakeholders participating in the project felt that the method used was largely transparent. It was seen as a natural way of working, in which interviewees felt they had control and influence over the content of the resulting framework. The iterative nature of the process was found to be advantageous, with stakeholders refining their own views and becoming more focused.

\section{INTRODUCTION}

We report on an empirical investigation of a recently developed method ${ }^{\prime}$ for CASE tool evaluation (Lundell and Lings 1999). The investigation was undertaken within Volvo IT, ${ }^{2}$ an organization of 2,500 workers responsible for the development and implementation of IT solutions for several large manufacturing plants in the automotive industry.

The company's initial motivation was to investigate the usage potential for a specific CASE tool, Visio $₫ 2000$ Enterprise Edition (Visio 1999), within the company's IS development life cycle. The company's Method Group also showed an initial interest in the method itself. The researchers' motivation was to investigate the method, and in particular its usability in terms of transferability, effectiveness, and scalability. This is in line with Fitzgerald (1996), who claims that one needs to "obtain empirical evidence of usability, which requires the method or technique to have been successfully applied to a nontrivial problem situation" (p. 12).

A critical analysis (Lundell and Lings 1997) of the ISO-standard for CASE tool evaluation (ISO 1995) revealed a lack of method support for the important task of establishing an evaluation framework to be used in a specific application of the standard (Lundell and Lings 1998). Lundell and Lings (2000) conducted an analysis of the literature on existing method support for CASE tool evaluation and identified pre-usage evaluation as "activities that take place before a tool being evaluated is in real use in a specific organizational setting" (p. 172). Such evaluation approaches aim to inform an organization before the (potential) selection of a CASE tool.

'To be fully presented in a Ph.D. Thesis, B. Lundell (in preparation).

${ }^{2}$ URL http://www.it.volvo.com (accessed February 26, 2001). 
Lundell and Lings identified four weaknesses in existing approaches to preusage evaluation, an important one being the lack of context dependence in evaluation frameworks defined a priori (see, for example, Chikofsky et al. 1992). They explored the potential for incorporating ideas from qualitative research, specifically grounded theory, to complement the standard (Lundell and Lings 1997, 1998) and have argued that their proposed method addressees all four weaknesses (Lundell and Lings 2000).

The method differs from existing general IS methods (e.g., Bubenko 1993; Galal and Paul 1999; Jayaratna 1994) in that it supports the often overlooked systematic exploration of technical issues.

In terms of method validity, as with qualitative methods in general, what matters from a specific application of the method is that the output is relevant rather than unique.

In section 2, We present the method and describe its transfer into the organizational setting. We then characterize the process of applying the method and give illustrative details from the evolving framework. Finally, based on postevaluation analysis with organizational stakeholders, we comment on the effectiveness of the method and summarize with a company experience of the method.

\section{ON THE METHOD AND ITS TRANSFER}

\subsection{The Method Used}

An inherent characteristic of the method is that it grounds an evaluation framework in the organizational setting. Previously developed frameworks can be used as (external) input data for the method, but they are afforded no special status. In particular, the method stresses the importance of a shared understanding among involved stakeholders for the emerging concepts in the framework and their interrelationships (Lundell and Lings 1997).

The major novelty in the method is its use of two distinct phases, with iteration between them(Figure 1). Briefly, the main role of phase one is to facilitate an in-depth understanding of need and develop a "rich" and relevant evaluation framework, whereas the main role of phase two (pilot evaluation) is to improve understanding of need, expand data sources, and introduce pragmatism into the framework

Application of the method is initiated with the selection of a number of data sources. Some of these will pre-exist, including organizational manuals, documentation of prior evaluation activities, and policy documents. Others will be generated; for example, transcripts of in-depth (open) interviews (phase one) with selected personnel. The data sources are analyzed with the goal of producing a set of inter-related concepts, with agreed interpretation, representing requirements for any CASE tool in the setting. 


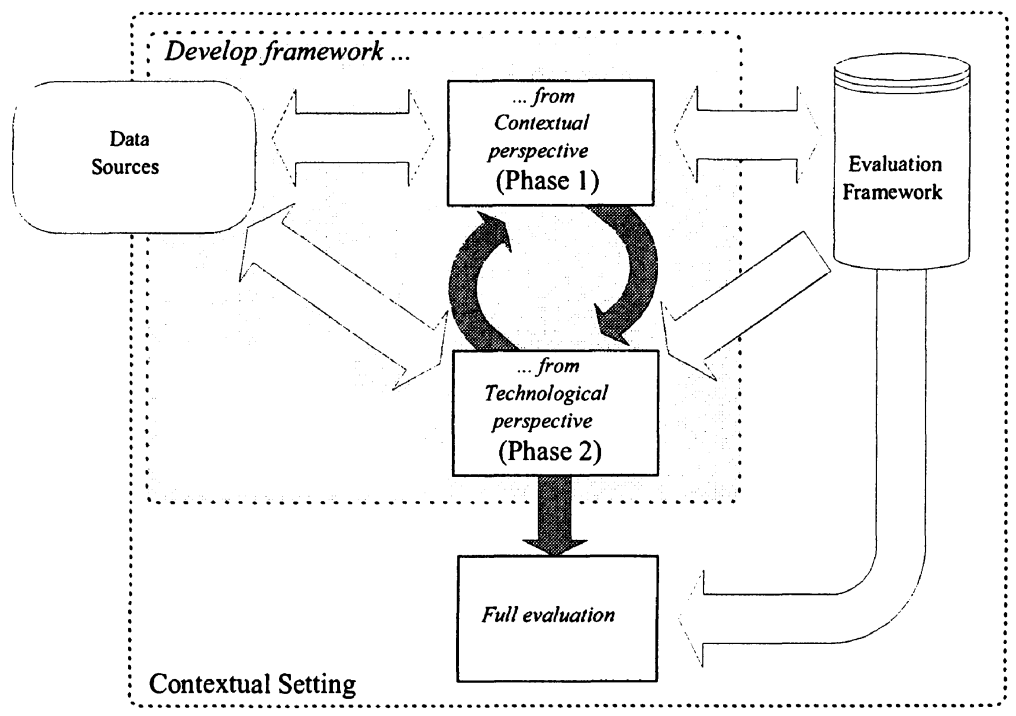

Figure 1. The Method Phases and Data Flow

In practice, the development of an evaluation framework is an evolutionary process involving data collection, analysis, and coding (Figure 1). These activities are not inherently sequential; each can affect (and trigger) the others so that, in essence, all activities are going on together. This characteristic is inherited from grounded theory, which informs the method (Glaser 1998).

It is important to the method that both pilot and full evaluations take place in the organizational setting in which any chosen tool would be used.

\subsection{On Method Transfer to Volvo IT}

Different management styles are evident between the two divisions involved in Volvo IT. The Methods Group in Gothenburg has responsibility for evaluating new technology and methods, thereby preparing other parts of the organization for adoption of suitable technologies. It is highly goal-oriented; management only gives direction concerning what is to be achieved and when. Given a critical mass of people, working procedures naturally emerge so as to facilitate fulfilment of the goals. In the Skövde division, which maintains and evolves systems that support operational business at a large engine factory, there is greater stress on economy and the ability to measure wherever and whenever possible, so that ultimately everything is predictable. 
A total of nine respondents were selected by the organization on the following premises: each had extensive knowledge of some part of the information systems life cycle; together, their knowledge covered the entire information systems development process; developers from both Skövde and Gothenburg were represented. In qualitative research, the field study would be characterized as using "elite interviews" (Marshall and Rossman 1999, p. 113).

\section{ON APPLYING THE METHOD}

\subsection{Developing an Initial Evaluation Framework}

Method application was initiated through an introductory e-mail describing the method, its purpose, interview outlines, and expected outcomes. The e-mail was followed up by booking interviews, over the phone or in person.

During interviewing, data collection was continually planned, using previous data collected. Occasionally leading questions were used, based on such data, and carefully framed to avoid rendering yes or no answers, as a means for guiding and/or fueling discussions. No recording devices ${ }^{3}$ were used; instead, field notes were shown openly, allowing stakeholders to correct and complement. This approach created an informal discussion climate and also avoided misinterpretations. The approach was unexpected and elicited a genuinely positive response.

Interviews (one to two hours each) were treated separately, and the material kept anonymous, thus avoiding issues of politics and personal prestige. The response from stakeholders vindicated this decision. Interview transcripts were, furthermore, sent to each stakeholder for comment and correction.

Transcripts were coded using a line-by-line concept extraction process. Codes were added as annotations and indicators were marked, creating a two column searchable structure in which codes (concepts ${ }^{4}$ ) identified their respective indicators. Complexity was significant but manageable. After removing duplicates and misspellings, a concept hierarchy emerged (see Table 1), which was later compiled into an evaluation framework (covering approximately 600 concepts).

${ }^{3}$ There are mixed views expressed in the literature, and although much research influenced by grounded theory reports that tape recording devices have been used, it is also claimed that the disadvantages from their use are more prominent than the potential benefits (e.g., Glaser 1998, p. 107).

${ }^{4} \mathrm{~A}$ concept is herein defined as a code together with its respective indicators. 
Table 1. Concept Structuring

\begin{tabular}{|l|l|}
\hline Concept category & Code generation \\
\hline Concept & Automate repetitive and structural coding \\
\hline Indicators & $\begin{array}{l}\text { Graphical representation handles the structural } \\
\text { coding-the developer may focus on solving the problem }\end{array}$ \\
$-\begin{array}{l}\text { Model driven so as to facilitate focus on helping to solve } \\
\text { problems and automatically generating skeleton source } \\
\text { code }\end{array}$ \\
\hline
\end{tabular}

'The top level identifies areas of concern; the second level identifies required CASE support; indicator(s) from transcripts support each concept (Rehbinder 2000, p. 150).

\subsection{Tool Exploration}

The method's second phase was initiated by exploring current technology support as represented by a state-of-the-art CASE tool (Visio 1999). The exploration was not initially constrained or guided by the framework, allowing new ideas concerning possible CASE support to arise. This "unrestrained" tool exploration provided both clarifications concerning framework demands as well as identification of many new and previously unrequested areas of tool support, guiding further investigations.

The focus then shifted to evaluating demands specified in the framework against current technology. The method user pursued investigation of seemingly unclear areas, focusing on code generation, database support, and components. Scope limitations were motivated by the study's focus on method transfer and effectiveness.

The chosen areas were explored in detail by investigating actual tool support for the selected concepts. The process generated data and insights both on supported and unsupported framework demands as well as on unrequested issues believed, by the method user, to have contextual relevance. Also pragmatic possibilities emerged, indicating alternative support or possible work-arounds relevant to the framework.

\subsection{Refining the Evaluation Framework}

Following tool exploration, another round of interviews was undertaken, using both the data and insights gathered along with the framework. Stakeholders also received a copy of the framework, allowing them to further comment and influence topics of discussion. 
Table 2. Framework Evolution (Refining a Stakeholder Concept from Table 1; Rehbinder 2000, p. 242)

\begin{tabular}{|l|l|}
\hline Concept & Automatic generation of system operation patterns \\
\hline Requirement & $\begin{array}{l}\text { CASE tools should support automatic generation of } \\
\text { structural coding including stubs, IDL, and trivial coding so } \\
\text { that models reflecting the system structure form the basis } \\
\text { for the system after which the developer may add } \\
\text { application logic. }\end{array}$ \\
\hline Indicators & $\begin{array}{l}\text { Model the pattern of operation that the system is } \\
\text { supposed to have and then have this implemented } \\
\text { complete with stubs and IDL so that only parts of the } \\
\text { business and application logic need be added. } \\
\text { CASE tools should generate stubs and trivial } \\
\text { programming from models describing the system and its } \\
\text { functionality }\end{array}$ \\
\hline
\end{tabular}

Stakeholder interest for this part of the study was surprisingly high. The method user was invited to further discussion on comments that stakeholders had made, providing a rich variety of new data. The method user interprets this as a success both concerning method transfer and relating to comprehensibility in the method's qualitative approach.

Transcripts were revised by stakeholders and then coded. The framework evolved, expanded, and increased in precision. For example, the concept automate repetitive and structural coding identified in the initial interviews evolved into two separate concepts: automate repetitive and structural coding and automatic generation of system operation patterns (see Table 2).

The use of phase two to evolve the framework was considered by stakeholders and the method user to have vital significance for the method's effectiveness.

\section{POST-EVALUATION OF THE METHOD APPLICATION}

To analyze the effectiveness of the method being used in this field-study, one of the authors undertook an analysis of stakeholder experience from this method application. From this analysis, ${ }^{5}$ which was undertaken as three open interviews following the field study, it is evident that the stakeholders' perception of this method and its application is positive. In the words of the interviewees:

${ }^{5}$ To be reported fully in a Ph.D. Thesis, B. Lundell (in preparation). 
- "It was fun to participate in the study and I'm willing to participate in forthcoming studies with the method" (Person 2)

- "The method can be adopted by our organization" (Person 1)

- "The goal for the method is easy to understand, even though the method itself was 'invisible' during the study" (Person 1)

Moreover, in addition to perceived positive experiences from participation in the working process during a method application, there was also a consensus ${ }^{6}$ among all interviewees during the post facto interviews that their ideas and knowledge concerning CASE tools had an influence on the content of the evaluation framework. For example:

- "I feel that my ideas concerning CASE tools were captured in the evaluation framework" (Person 2)

- "It [the content of the framework] resulted in a good focus" (Person 3)

The iterative nature of the method, with partial deliverables and some time between them, was considered effective, as illustrated by one interviewee:

- "At the second interview, the customer starts from a better basis. What happens in people's minds over time implies that we achieve a better basis to start with" (Person 3)

There was also the perception of a significant technical demand, which any method user needs to fulfil, in order to be capable of undertaking a CASE tool exploration that can provide useful data concerning the tools for exposure to the organizational stakeholders. This issue was discussed during one interview and the necessary pre-knowledge was stressed as follows:

- "I think that the method user should have a fairly good knowledge of what kinds of things CASE tools can do. Of course, there is a balance. With too much knowledge, it would obviously steer the method user towards the experience he/she has at that time" (Person 1)

\section{SUMMARY}

The motivation for this field study was to investigate the effectiveness of a proposed method for CASE tool evaluation within a large company. Its degree

${ }^{6} \mathrm{All}$ three post facto interviews confirmed this. 
of success must, therefore, be judged from a company perspective. The company experience of the method has been summarized by a senior manager in the Method Group in the following way:

This method is very useful, if entered in the mood of having to decide between equal tools where the [vendor] company structures have little or no impact on the evaluation. If significant procedures for evaluating [vendors] and their impact on future trends are added, a versatile method for evaluation is created. This is the best I have seen so far, and I have seen a lot.

\section{ACKNOWLEDGMENTS}

The authors are indebted to the participants in the study at Volvo IT, the Volvo IT organization for making this study possible, and in particular the two divisions in which this study was undertaken. We would also like to thank the many other Volvo IT stakeholders that contributed to the project. Finally, we would like to thank the participants in previous studies with the method, and their organizations, for providing valuable input toward its further development.

\section{REFERENCES}

Bubenko, J.A. Jr. "Extending the Scope of Information Modeling," in Fourth International Workshop on the Deductive Approach to Information Systems and Databases, A. Olivé (ed.), Lloret de Mar, Catalonia, September 20-22, 1993, pp. 73-93.

Chikofsky, E. J., Martin, D. E., and Chang, H. “Assessing the State of Tool Assessment," IEEE Software (9:3), 1992, pp. 18-21.

Fitzgerald, B. "Formalized Systems Development Methodologies: A Critical Perspective," Information Systems Journal (6:1), 1996, pp. 3-23.

Galal, G. H., and Paul, R. J. "A Qualitative Scenario Approach to Managing Evolving Requirements," Requirements Engineering (4:2), 1999, pp. 92-102.

Glaser, B. G. Doing Grounded Theory: Issues and Discussions, Mill Valley, CA: Sociology Press, 1998.

ISO. "Information Technology: Guideline for Evaluation and Selection of CASE Tools," International Organization for Standardization/International Electrotechnical Commission ISO/IEC JTC1/SC7/WG3, ISO/IEC 14102:1995(E), 1995.

Jayaratna, N. Understanding and Evaluating Methodologies-NIMSAD: A Systemic Framework, London: McGraw-Hill, 1994.

Lundell, B., and Lings, B. "An Empirical Approach to the Evaluation of CASE Tools: Method Experiences and Reflection," in Proceedings of the Third CAiSE/IFIP8.1 International Workshop on Evaluation of Modeling Methods in System Analysis and Design (EMMSAD'98), K. Siau (ed.), Lincoln, NE: University of Nebraska-Lincoln Press, 1998, pp. $\mathrm{N}: 1-12$.

Lundell, B., and Lings, B. "Evaluation and Selection of CASE Tools Within the ISO Framework: Qualitative Issues," in ACIS'97: Proceedings of the Eighth Australasian Conference on 
Information Systems, D. J. Sutton (ed.), School of Information Systems, University of South Australia, Adelaide, South Australia, Australia, September 29-October 2, 1997, pp. 203-214. Lundell, B., and Lings, B. "On Method Support for Developing Pre-Usage Evaluation Frameworks for CASE Tools," in Systems Development Methods for Databases, Enterprise Modeling, and Workflow Management, W. Wojtkowski, W. G. Wojtkowski, S. Wrycza, and J. Zupancic, (eds.), New York: Kluwer Academic/Plenum Publishers, 2000, pp. 169-181. Lundell, B., and Lings, B. "Validating Transfer of a Method for the Development of Evaluation Frameworks," in Proceedings of the Sixth European Conference on Information Technology Evaluation, A. Brown and D. Remenyi (eds.), Brunell University, Uxbridge, UK, November 4-5, 1999, pp. 255-263.

Marshall, C., and Rossman, G. B. Designing Qualitative Research ( $3^{\text {rd }}$ Edition), Thousand Oaks, CA: Sage Publications, 1999.

Rehbinder, A. On Applying a Method for Developing Context Dependent CASE Tool Evaluation Frameworks, Master's Thesis, Department of Computer Science, University of Skövde, Skövde, Sweden (HS-IDA-MD-00-012), 1999.

Visio. Visio $₫ 2000$ Enterprise Edition: User Guide, Seattle, WA: Visio Corporation, November 1999.

\section{About the Authors}

Adam Rehbinder holds an M.Sc. from the University of Skövde and has recently left the University of Skövde to work as an IT Consultant at Rehbinder $\&$ Co. Konsulter AB. Adam can be reached by e-mail at rehbindera@ hotmail.com.

Brian Lings has been a lecturer in the Department of Computer Science at the University of Exeter since 1982. His research interests concern the development of tools and methods for the successful exploitation of database technology in complex information sharing domains. Much of his work is conducted in collaboration with colleagues at the University of Skövde, Sweden. Brian can be reached by e-mail at B.J.Lings@exeter.ac.uk.

Björn Lundell was awarded an M.Sc. (1991) in Computer Science from University of Skövde, Sweden, where he is currently a staff member and studying for a doctorate at the University of Exeter, UK. His current research activities center around CASE technology and associated method support for CASE tool evaluation and in particular for the task of establishing evaluation frameworks. Björn can be reached by e-mail at bjorn@ida.his.se.

Runo Burman is a senior Methods Engineer at the Common Skills and Method, Volvo IT, Göteborg. Runo can be reached by e-mail at runo.burman@ volvo.com.

Anette Nilsson is a project manager at Volvo IT, Skövde. Anette can be reached by e-mail at anette.em.nilsson@volvo.com. 